\title{
Baselining the Whole-of-Force Capability and Capacity of the Australian Defence Force
}

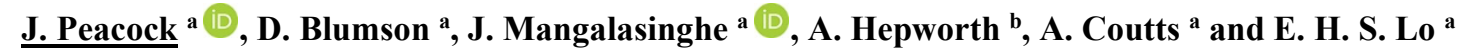 \\ aJoint and Operations Analysis Division, Defence Science and Technology Group, Australian Defence Force, \\ ${ }^{b}$ Land Warfare Lab, Army Headquarters, Department of Defence \\ Email:james.peacock@dst.defence.gov.au
}

\begin{abstract}
This paper outlines an analytical approach taken to baseline the whole-of-force capability and capacity of the objective Australian Defence Force (ADF) (i.e. the projected force). This was achieved by capturing structured subjective assessments from subject matter experts (SMEs) drawn from across Defence. Approximately 200 SMEs participated in the assessments and were split into syndicates of 20 - 30 participants. Each syndicate completed a series of tasks to identify their subjective assessment of the ADF in eight Australian Capability Context Scenario's (ACCS). This was performed for both an individual ACCS and concurrent ACCS over four time epochs. The following tasks were performed by each syndicate for an individual ACCS: (i) Develop a Concept of Operations (CONOPS) specific to the ACCS; (ii) build a force model, comprising types and quantities of Defence Elements (e.g. platform such as F-35A Joint Strike Fighter or Infantry Fighting Battallion), that was constrained by the objective Australian Defence Force inventory; and (iii) perform a structured subjective assessment to determine the performance of the force model to realise the CONOPS. For concurrency, where a syndicate was assigned multiple (two to four) ACCS', the outputs from the individual ACCS were provided and their task was to rationalise the multiple force models to be constrained by a singular ADF inventory. Each syndicate performed a reassessment of the revised force models to realise each individual concept of operation. The outcome of these tasks enabled the identification of high and low performance of the ADF in a specific context (i.e. capability and capacity of Defence Elements specific to a scenario). This paper holds that a judgement-based form of decision support modelling (a form of multi-criteria decision making) provided appropriate structured analytical support to some of the most critical decisions to be made by the Department of Defence within the time-constraints imposed.
\end{abstract}

Keywords: Force structure plans, Defence capability assessment, Objective Force, Military force performance 


\section{INTRODUCTION}

Force Structure Plan 2019 was the designated campaign to review and update the Integrated Investment Program over the forward estimates, inline with force design doctrine (McKenna \& McKay, 2017). The Force Structure Plan contained seven constituent activities, each addressing a distinct line of effort. The aim of Activity 5 was to baseline the capability and capacity of the objective whole-of-Australian Defence Force (ADF) (i.e. the projected force in the future) to achieve Government directed Defence Missions. This paper outlines the methods used to perform this baselining activity.

The whole-of-force capacity and capability was to be tested in its ability to succeed in Australian Capability Context Scenarios (ACCS). Department of Defence (2010) describe the ACCS as possible circumstances under which the future joint force might be employed. Each individual ACCS describe a geographic context under which the users (i.e. the participants in the present study) inject a blue force to overcome the red force. The blue and red force is not only constrained to military powers, but can also involve other actors such as civilians and non-governmental actors. Implementing a number of ACCS ensures a broad range or spectrum of plausible and illustrative future contingencies in terms of threat and duration.

The complexity of the scenario-based problems and the number of dimensions across whole-of-force precluded detailed planning, bespoke simulation and traditional wargaming as feasible methods. Consequently, a decision support model, that traded-off fidelity for feasibility and relied on structured elicitation of subject matter experts (Wijnmalen \& Curtis, 2013), was employed. Similar to the role of seminar wargaming in related strategic problems (Davis, 2016), this approach provided agility, creativity and transparency, breadth to cover the whole-of-force, and human phenomena, allowing the conclusions drawn from this experiment to aid strategic decision making.

In line with this approach, a collaborative decision support model was designed to assess the capability and capacity of the ADF over different timeframes and scenarios. Structured subjective observations were captured from approximately 200 subject matter experts (SMEs) under eight ACCS across four time epochs. The SMEs, from different service domains, were divided into syndicates of approximately $20-30$ participants. Each syndicate developed a concept of operations (CONOPS) (i.e. a strategic plan for the scenario) and force model (i.e. assignment of types and quantities of platforms, units, etc.) to realise both an individual ACCS and multiple ACCS' occurring concurrently. The developed force models were constrained by the objective ADF's inventory, which revealed gaps and limitations in the capability and capacity of the ADF. The ability of the ADF to succeed in each ACCS was measured using a structured participant assessment. The conceptual framework for the study was that low or high ADF performance would be determined by the participants' perceptions of the capacity and capability of the objective ADF within the defined scenarios.

\section{ASSESSMENT ACTIVITY DESIGN}

A four-step process was conducted over a two-week period to baseline the ADF and was developed around the flowchart for planning Defence experiments and campaigns (The Technical Cooperation Program, 2006):

1. Abbreviated military appreciation to develop a CONOPS and a force model to meet an individual ACCS (week 1).

2. Capability assessment of the force model to meet the concept of operation (week 1).

3. Force model rationalisation across concurrent ACCS' (week 2).

4. Capability reassessment of force models under concurrent ACCS' (week 2).

The abbreviated military appreciation (step 1) required syndicates to study and summarise a given threat scenario and develop a CONOPS. A force model comprising Force Packages and Defence Elements was developed to realise the CONOPS. Step 2 required syndicates to assess the performance of the force model to achieve the CONOPS. Steps 1 and 2 were performed across four time epochs.

In Step 3, participants examined a set of concurrent scenarios, where two to four ACCS occurred simultaneously. The CONOPS and force models developed during the first week were provided to participants; step 3 was to rationalise the multiple force models to be constrained by a single ADF inventory. Where an over-allocation was identified, participants were asked to explore either alternative Defence Elements in the inventory to achieve the same effect or to share Defence Elements within or across scenarios. Step 4 involved reassessing the performance of the force models to achieve each individual scenario. It was hypothesised that performance of the force models would reduce under concurrency due to the constrained number of Defence Elements supporting multiple operations. 


\subsection{Defence Elements, Force Packages and Force models}

Defence Elements defined a unit of an ADF capability. Examples of Defence Elements include platforms such as the F-35A Joint Strike Fighter and Hobart-class destroyer, units such as motorised infantry battalion and enterprise enablers such as Collective Training. Defence Elements represented the highest level of resolution in the employed capability taxonomy.

Force Packages were a mandated construct for the assessment and are defined as context independent, temporal building blocks that account for Force-in-Being and objective ADF capabilities including enablers. Force Packages were prepopulated with types and numbers of Defence Elements for each explored epoch. Defence Element types within a singular Force Package were grouped based on similar functional tasks, for example, the EA-18G Growler existing within the same Force Package as the F-35A Joint Strike Fighter and F/A-18F Super Hornet as they complement each other (Royal Australian Air Force, 2019). This data was prepopulated into the force model builder tool as described in Section 3, to enable syndicates to compose force models.

\subsection{Force model Performance: Joint Capability Effects (JCE), Joint Warfighting Functions (JWF), and Overall Mission Success}

Three levels of assessment were used to characterise the performance of the force models across each of the four time epochs to realise the CONOPS (in order of highest to lowest) for each ACCS: (i) Overall Mission Success, (ii) JCEs, and (iii) JWFs. Likert scales (Table 1) (Leedy \& Ormrod, 2005) were used to structure the subjective ratings of performance at each of the three levels of assessment. In week 1, for the individual ACCS, the assessment was performed across all three levels. In week 2, for performance reassessment under concurrency, the assessment was performed for overall mission success and JCEs

Table 1. Five-point Likert scale applied throughout assessment.

\begin{tabular}{ll}
\hline Quantitative score & Qualitative definition \\
\hline 5 & Significantly overachieves the requirements \\
4 & Overachieves the requirements \\
3 & Achieves the requirements \\
2 & Underachieves the requirements \\
1 & Significantly underachieves the requirements \\
\hline
\end{tabular}

The sponsor mandated JCEs as the primary lens for assessing the ability of generated force models to achieve desired operational level effects. Joint Capability Effects represent the ability to perform primary (e.g. Air Control, Strike and etc.) and secondary (e.g. Force Projection and Lift, Sustain the Force, etc.) functions. The JCE construct sought to establish a link between Strategy and delivery while translating temporal strategic priorities to operational responses using Force Packages. The JCEs were:

1. Situational Awareness and Intelligence

2. Command, Control and Communications (C3) and Force Information Assurance

3. Strike

4. Air Control

5. Sea Control

6. Land Control

7. Cyber Control

8. Space Access and Control

9. Support to Civil Authorities

10. Force Projection and Lift

11. Enterprise Force Generation

12. Sustain the Force

13. Influence and Engagement

14. Homeland Security.

Many of the JCEs are necessarily high level and encompass many warfighting functions - for example Land Control, Air Control and Sea Control - and therefore do not have a clear mapping to changes in deployed capabilities. Consequently a two-tiered approach of assessment was performed to elicit the participant's assessment of the force model to achieve each JCE. This was achieved by providing an assessment for each of the six JWFs (Table 2) within the context of the JCE. These primary assessments then informed the assessment of the JCE (Figure 1, which uses fictitious data) through an aggregated score (Equation 1). For each relevant JWF, participants were asked how well they believed the designed force model achieved that JWF, within the context of the JCE in question. If a JWF was deemed irrelevant, such as the ability to perform Force Application for Sustain the Force JCE, that assessment did not contribute to the aggregated JCE score. In some instances a JWF was deemed to be more relevant than others, and the aggregated JCE score was weighted accordingly. 
Peacock et al., Baselining the Whole-of-Force Capability and Capacity of the Australian Defence Force

Table 2. Joint Warfighting Functions (Department of Defence, 2012).

\begin{tabular}{ll}
\hline Joint Warfighting Function & Definition \\
\hline Command & $\begin{array}{l}\text { The overarching warfighting function that enables all others. Decision } \\
\text { superiority is a central aspect of command. }\end{array}$ \\
Situational Understanding & $\begin{array}{l}\text { Essential prerequisite for the conduct of campaigns and operations, } \\
\text { facilitating decision superiority. }\end{array}$ \\
Force Projection & $\begin{array}{l}\text { The ability to project force to exploit the operational environment and } \\
\text { deny it to an adversary. Is inextricably linked with manoeuvre. }\end{array}$ \\
Force Application & $\begin{array}{l}\text { Combination of manoeuvre and offensive action. } \\
\text { Force Protection }\end{array}$ \\
Force Generation and & $\begin{array}{l}\text { To ensure the physical integrity and morale of the fighting force. } \\
\text { Sustainment }\end{array}$
\end{tabular}

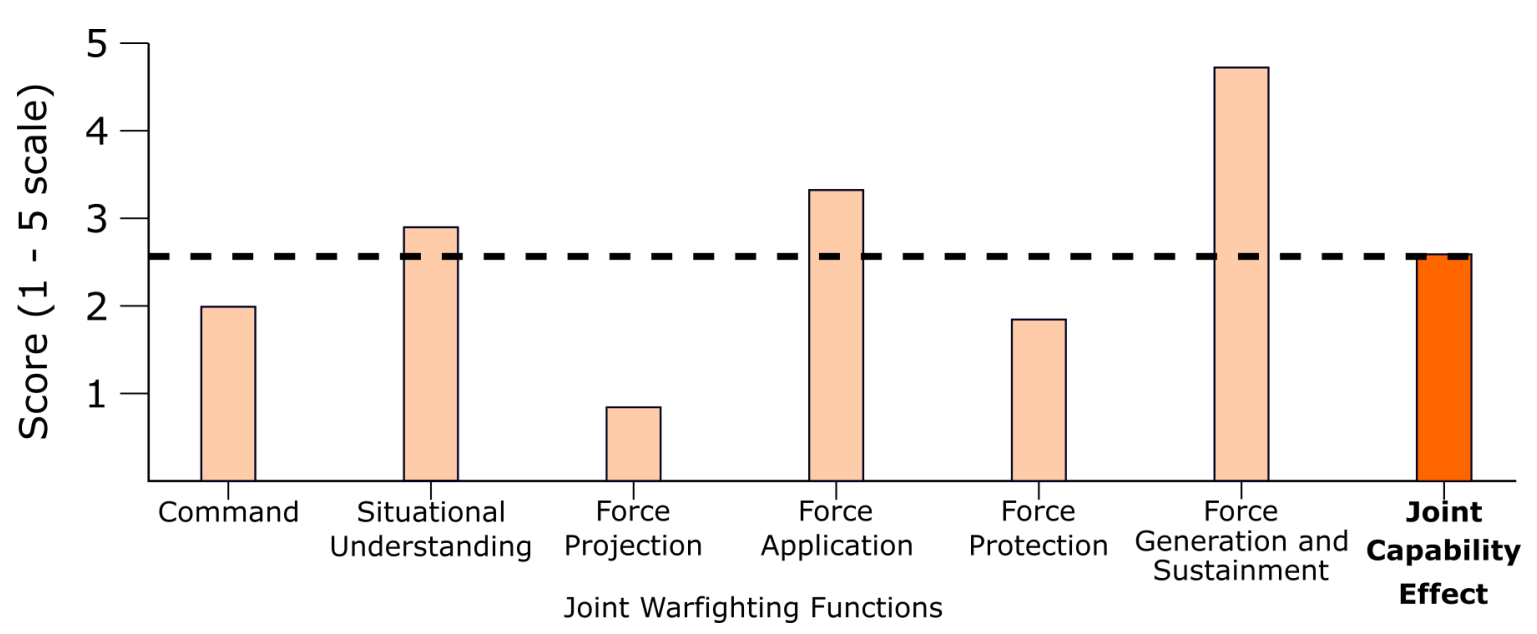

Figure 1. JCE score from aggregated JWF scores. The data contained in this figure is fictitious.

$$
\sqrt{\frac{\sum S_{i}^{2} R_{i}^{2}}{\sum R_{i}^{2}}} \text { where } S_{i} \text { is the JWF score and } R_{i} \text { is its relevance }
$$

The aggregation of JWF to JCE (Equation 1) was a combination of weighted mean and quadratic mean. The weighted mean provided greater weighting to JWFs of higher relevance to the estimated JCE score. The quadratic mean provided a greater weighting to the product of both high score and high relevance to the estimated JCE score. In combination, JWFs that were of high relevance and/or of high scores had a larger influence on the estimated JCE score.

Following assessment of JWFs within an individual JCE, participants had the ability to adjust the aggregated JCE score. Participants were asked: "How would the designed force model achieve the Sea Control JCE?" Participants adjusted the aggregated score, provided they considered the aggregation as a means of providing structure and a basis for their subjective scores to support the traceability of collected data.

Assessment of overall Mission Success was performed using a similar two-tiered structured approach. The assessment of each JCE was provided to the participants to inform their assessment of overall mission success using a 7-point Likert scale (Leedy \& Ormrod, 2005).

Assessment data were captured at the individual and group level across different stages of analysis. JWFs were captured at the individual level. Individual JWF scores were aggregated using a weighted function, similar to Equation 1. The weighting was achieved by participants providing a level of expertise with values of 1,2 , and 3 for low, medium and high expertise, respectively, within the context of each JWF and JCE. Data for the refinement of JCE and the subsequent overall mission success were captured at the group level.

To measure uncertainty in the performance of each force model to achieve the CONOPS, the assessment was performed at three levels: most likely, worst-case, and best-case. Rather than calculating uncertainty through 
inferential statistical methods, such as standard deviation or confidence interval, and because assessments at the JCE and overall mission success were performed at a group level, uncertainty in the performance of the force model was by participants' perceptions. Most-likely, worst-case and best-case were interpreted by the participants in the uncertainty of how the campaign might unfold: the worst-case score was under the anticipation of a large number of casualties or platform degradation, and the best-case score was made under the anticipation of the low casualties or platform degradation.

\section{DATA CAPTURE TOOLS}

Three tools were developed within Microsoft Excel: the force model builder, force assessment tool, and gaps, limitations, opportunities and risk Tool.

\subsection{Force model builder tool}

The force model builder tool supported participants to build a force model across four time epochs that was constrained by the ADF's inventory using the construct of Force Packages. Further, the tool supported making a connection of Force Packages and Defence Elements to JCEs. This additional step supported traceability of poor assessment scores to Force Packages and their contents. The ultimate goal of this tool was to identify the type and number of Defence Elements required to realise the CONOPS.

After developing the CONOPS, participants recorded the relevancy of each JCE (highly relevant, relevant, or not relevant). Participants then selected Force Packages necessary to realise the CONOPS within the context of each JCE (Figure 2).

\begin{tabular}{|c|c|}
\hline JCE3 - Strike & Strategic Strike Assessment \\
Relevance of JCE to mission: & Air Find-Fix-Track \\
\cline { 2 - 2 } RELEVANT & Air Base / Port Operations \\
& Operational C2 \\
\hline & Airborne Operations \\
\hline
\end{tabular}

Figure 2. Selection of Force Packages to achieve JCEs. The data contained in this figure is fictitious.

In a second worksheet, the selected Force Packages and associated Defence Elements were displayed (Figure 3). Participants then, within the context of each Force Package, identified the quantities of each Defence Element. This was a two-step process. First, participants recorded the quantitiy of required Defence Elements. This captured the demand of the Defence Elements needed for the concept of operation within the context of the ACCS. Secondly, participants refined their quantities by making an allocation that was constrained by the ADF's inventory. The relationship between Force Packages and Defence Elements was "many-to-many". This meant a Defence Element was listed multiple times if it existed in multiple Force Packages. To avoid double counting Defence Element quantities, there were two forms of recording the metric - dedicated and shared. The quantity within the dedicated cell was summed for each instance the Defence Element was listed within the force model. For the shared cell, only the maximum of all instances of the Defence Element was counted. This allowed for the instance of a Defence Element that existed in multiple Force Packages supporting multiple operations/functional tasks, to only be counted once but still be recorded as contributing to multiple Force Packages. Under concurrency, participants made the allocation of Defence Elements and their quantities to each ACCS while being constrained by a single ADF inventory.

\begin{tabular}{|c|c|c|c|c|c|c|c|c|c|}
\hline \multirow{2}{*}{$\begin{array}{c}\text { Force Package } \\
\text { (pre-filled from JCE }\end{array}$} & \multirow{2}{*}{$\begin{array}{l}\text { Defence } \\
\text { elements }\end{array}$} & \multicolumn{2}{|c|}{ Required } & \multicolumn{2}{|c|}{ Allocated } & \multicolumn{3}{|c|}{ ADF inventory } & \multirow[b]{2}{*}{ Notes } \\
\hline & & Dedicated & Shared & Dedicated & Shared & Total & Allocated elsewhere & Remaining & \\
\hline \multirow{3}{*}{$\begin{array}{l}\text { Airborne } \\
\text { Operations }\end{array}$} & Platform 2 & 6 & 0 & 4 & 0 & 4 & 0 & 0 & \multirow{3}{*}{$\begin{array}{l}\text { Gap mitigated by Platform } 3 \\
\text { Gap: insufficient capacity }\end{array}$} \\
\hline & Platform 4 & 10 & 0 & 2 & 0 & 2 & 0 & 0 & \\
\hline & Platform 5 & 2 & 0 & 2 & 0 & 1 & 0 & -1 & \\
\hline
\end{tabular}

Figure 3. Selection of Force Packages to achieve JCEs. The data contained in this figure is fictitious.

\subsection{Force Assessment and Concurrency Reassessment Tools}

The Force Assessment Tool captured and aggregated assessment scores across the three tiers of analysis. The assessment process began with individual scores for each JWF within the context of a singular Joint Capabilty Effect. The best-case, most likely and worst-case scores were captured from each individual and were 
aggregated to indicate the JCE score. Participants had the opportunity to then provide a refinement of this overall score. A similar process was conducted to identify overall mission success, as informed by the JCE scores. Under concurrency (week 2 data capture), participants viewed the assessment scores for each individual ACCS at the JCE and overall mission success levels, where they made adjustments to scores based on the revised force model (more commonly a reduced quantity of Defence Elements for each model).

\subsection{Gaps, Limitations, Opportunities and Risks Tool}

Crucial to the understanding of the assessment scores was the identifitication of the gaps, limitations, opportunties and risks of the designed force model and CONOPS for each ACCS. While there were opportunities for participants to provide qualitative explanations within the force model builder tool and force assessment tool, these comments were often unstructured, lacked important meta-data (such as participant identification number, Defence Element, etc.) and the opportunity for noting these comments occurred during a limited time period (e.g. during assessment time). Subsequently, a tool was developed to capture qualitative comments in a structured format (to facilitate analysis) and be readily available.

\section{DISCUSSION}

\subsection{Capability Comparison Graphs}

Results from quantitatively assessing the ADF through the lens of a JCE yielded capability comparison graphs (Figure 4). Further merging of data gathered from the gaps, limitations, risks and opportunities tool, threat summary and CONOPS provides explanatory evidence to interpret the graphs. To illustrate, four hypothetical assessments on four JCEs are analysed based on the "most-likely" case of a fictitious scenario with friendly Blue against opposing Red Forces:

a. For all four epochs, Blue Force is able to overachieve Land Control

b. After achieving Strike in epochs 1 and 2, advancements in enemy Anti-Access Area Denial (A2AD) capability in later epochs leads to underachievement of the JCE

c. Planned withdrawal from service of Blue Fighter Jets in epoch 3, coupled with delays to the replacement Defence Element, leads to reduced Air Control capability until epoch 4

d. Blue Force maritime investments leads to improved ability to achieve Sea Control in later epochs



Figure 4. Illustrative capability comparison graphs for selected JCEs over four time epochs. Note; data were transformed from the 1 to 5 to -2 to +2 on the Likert scale. The data contained in this figure is fictitious.

The quantitative outputs across JCEs, time epochs, Defence Elements and Force Packages enabled decision makers to make informed decisions on capability acquisitions. For example, the fictitious scores indicate there may exist opportunities for divestment in Land Control. In Air Control, setting the withdrawal date of Blue Fighter Jets to overlap with the introduction of its replacement capability will avoid a capability gap in Air Control. Using JCEs as the metrics, material acquisition was not limited to acquiring the latest iteration of a platform replacement, rather the focus was on raising deficiencies in operational-level effects, whereby employing a new operating concept may be equally effective. 


\subsection{JCEs and JWFs}

Joint Warfighting Functions are the necessary requirements in the battlespace that enable successful achievement of an outcome, which may be a JCE. The JWFs were used to infer initial JCE scores for consideration and ratification by participants. This was implemented by participants rating the performance of the JWFs within the context of a JCE. Participant feedback during the assessment identified the poor mapping between JWFs with some JCEs. The mapping between JWFs and some JCEs - such as Sea Control, Land Control and Strike - was appropriate. Participants were able to successfully use the JWFs to support the assessment of these JCEs. But, for JCEs such as Force Projection and Lift, the mapping between JWFs was not appropriate as its reliance on the Force Projection JWF has a greater significance than a reliance on the other JWFs. Participants identified that the mapping in similar instances was not useful. For future work, it is recommended that assessments are performed with JCEs that exist at functionally separated levels.

\subsection{Limitations to analytical approach}

The scale and complexity of the analytical problem was beyond the ability of existing processes and tools. The activity examined the ability of the ADF over four time periods to conduct eight large scale scenarios - both individually and concurrently with other scenarios. The complexity of the ACCS and the number of dimensions involved precluded detailed planning, bespoke simulation and traditional wargaming as feasible methods. Instead, a structured decision support approach and associated tool set were developed to collect contextualised subjective data from SMEs. Such an approach essentially traded-off validity - primarily in the ability to properly account for biases in the activity - for the viability of providing a high-level overview of the analytical problem. From the results, areas of concern could be highlighted for more detailed analysis (Au, Hoek, \& Lo, 2018). The challenge is to assess model validity in the context of its purpose (Barlas, 1996).

\section{CONCLUSIONS}

This paper outlined an analytical approach used to baseline the whole-of-force assessment of the ADF. Here we detailed how structured subjective data were captured to identify the capability and capacity of the wholeof-force. These data provide an evidence base that supports the decision making of future investments.

\section{ACKNOWLEDGEMENTS}

The authors would like to acknowledge the contribution of Matthew Richmond, Richard Fleming, Peter Hoek, Ralph Gailis, Brett Laboo, Mark Tutton, Fred Bowden, Phuong La, Frank Lui, Niem Tri, and Tim Heseltine.

\section{REFERENCES}

Au, T. A., Hoek, P. J., \& Lo, E. H. S. (2018). Combat Analysis of Joint Force Options using Agent-Based Simulation. IEEE: Proceedings of the Military Communications and Information Systems Conference.

Barlas, Y. (1996). Formal aspects of model validity and validation in system dynamics. System Dynamics Review, 12(3), 183-210.

Davis, P. K. (2016). Capabilities for Joint Analysis in the Department of Defense - Rethinking Support for Strategic Analysis. Santa Monica, California, USA: RAND Corporation.

Department of Defence. (2010). The Strategy Framework. Canberra, ACT: Defence Publishing Service.

Department of Defence. (2012). ADDP 3.0: Campaigns and Operations. In Operations Series.

Leedy, P. D., \& Ormrod, J. E. (2005). Planning and Design.Practical Research (Eighth ed.): Pearson Education.

McKenna, T., \& McKay, T. (2017). Australia's Joint Approach: Past, Present and Future. In Joint Studies Paper Series No. 1: Defence Publishing Service.

Royal Australian Air Force. (2019). EA-18G Growler. Retrieved from https://www.airforce.gov.au/technology/aircraft/strike/ea-18g-growler

The Technical Cooperation Program. (2006). Guide for Understanding and Implementing Defense Experimentation (GUIDEx). In.

Wijnmalen, D., \& Curtis, N. J. (2013). A code of best practice for judgement-based operational research. $O R$ Insight, 26(4), 291-308. 